FORMATION Formation emploi

Revue française de sciences sociales

113 | janvier-mars 2011

La flexicurité à l'aune de l'approche par les capacités

\title{
Les effets de la formation diffèrent selon les parcours sur le marché du travail : l'exemple catalan
}

The effects of training differ depending on pathway through the employment market: the example of Catalonia

Die unterschiedlichen Auswirkungen der Ausbildung je nach Berufsweg auf dem Arbeitsmarkt : ein katalanisches Beispiel

Los efectos de la formación difieren según los itinerarios en el mercado de trabajo : el ejemplo catalán

Martí López-Andreu et Joan Miquel Verd

\section{OpenEdition}

\section{Journals}

Édition électronique

URL : http://journals.openedition.org/formationemploi/3249

DOI : $10.4000 /$ formationemploi.3249

ISSN : 2107-0946

Éditeur

La Documentation française

Édition imprimée

Date de publication : 1 mars 2011

Pagination : 63-79

ISSN : 0759-6340

Référence électronique

Martí López-Andreu et Joan Miquel Verd, « Les effets de la formation diffèrent selon les parcours sur le marché du travail : I'exemple catalan », Formation emploi [En ligne], 113 | janvier-mars 2011, mis en ligne le 10 avril 2013, consulté le 30 octobre 2020. URL : http://journals.openedition.org/ formationemploi/3249; DOI : https://doi.org/10.4000/formationemploi.3249 


\section{DOSSIER}

\section{Les effets de la formation diffèrent selon les parcours sur le marché du travail : l'exemple catalan}

Martí López-Andreu, Joan Miquel Verd*

Les différents types de formation peinent à contrebalancer certaines inerties du marché de l'emploi. Ce sont les circonstances de l'utilisation de la formation, et non pas son utilisation en elle-même, qui confèrent son efficacité à la formation.

Des politiques salariales tendant à la libéralisation et à la flexibilisation se sont développées à grande échelle dans toute l'Europe, au cours de ces vingt dernières années. Comme l'a noté Alonso (2007, Chap. 3), d'une part ce type d'intervention implique des mesures destinées à favoriser un recours massif à la force de travail, en réduisant son coût, et, d'autre part, il impulse (et finance) des formes d'embauche flexibles, en créant un nombre croissant de modalités contractuelles.

L'Espagne n'a pas fait exception à cette règle européenne, bien au contraire ${ }^{1}$. Les forts taux de chômage qu'a connus ce pays, au début des années 80, ont conduit à réformer en profondeur la législation du travail et à inventer de nouvelles formes de contrat, plus flexibles. C'est sur la base de ces réformes que s'est développé, à partir de 1985, le contrat à durée déterminée. Le taux de travailleurs recrutés en CDD (contrat à durée déterminée) a très vite atteint des

\footnotetext{
${ }^{1}$ Le manque de flexibilité du marché du travail en Espagne a été, et demeure, un argument récurrent lorsqu'il s'agit d'expliquer le taux élevé de chômage en Espagne par rapport à la plupart des pays
} voisins ( $c f$. Recio, 1997, pp. 169-171).
* Martí López-Andreu, diplômé en sociologie, termine sa thèse de doctorat. Professeur associé, il est chercheur au département de sociologie de I'Université Autonome de Barcelone (UAB) au Centre d'études sociologique sur la vie quotidienne et le travail (QUIT). Ses recherches portent sur les transformations et les changements induits par la flexibilisation du travail au niveau de l'entreprise et au sein des politiques sociales et du travail. Elles traitent également de leurs effets sur les parcours sur le marché du travail, et se centrent sur les dynamiques inégalitaires. Publications récentes : López Andreu M. (2008): "Les reformes de la prestació d'atur a l'Estat Espanyol en el marc de la Estratègia Europea d'Ocupació", en Papers. Revista de Sociología, 88, pp. 11-30; J.M.Verd, J. Vero y M. López Andreu (2009), "Trayectorias laborales y enfoque de las capacidades. Elementos para una evaluación longitudinal de las políticas de protección social", en Sociología del Trabajo, 67, pp. 127-150.

Joan-Miquel Verd est diplômé en sciences politiques et sociologie, et en sciences économiques et de gestion. 
Professeur titulaire au département de sociologie de I'Université Autonome de Barcelone (UAB), il est rattaché au Centre d'études sociologique sur la vie quotidienne et le travail (QUIT). Sur le plan méthodologique, ses recherches portent sur l'analyse biographique et l'étude des réseaux sociaux. Sur le plan théorique, il s'intéresse à la relation formation-emploi, les compétences et le lien entre protection sociale et emploi. Publications récentes : P. Bartelheimer, N. Moncel, J. M. Verd y J. Vero (2009) "Towards Analysing Individual Working Lives in a Resources/Capabilities Perspective", en NET. Doc, 50, pp. $21-50$; J. M.Verd, J.Vero, y M. López (2009), "Trayectorias laborales y enfoque de las capacidades. Elementos para una evaluación longitudinal de las políticas de protección social", en Sociología del Trabajo, 67, pp. 127-150 ; A. Kuznik, J. M. Verd (2010) "Investigating Real Work Situations in Translation Agencies. Work Content and Its Components", en Hermes. Journal of Language and Communication Studies, 44, pp. 25-44.

proportions très importantes. En 1987, 15,6 \% des travailleurs travaillaient sous ce type de contrat et, en 1992, ce pourcentage atteignait 33,5\% (Santos et Poveda, 2002, p. 265). Aujourd'hui, cette situation n'a pas fondamentalement varié $(25,08 \%$ selon les résultats de l'Enquête de Population Active du dernier trimestre de 2009), au point que certains auteurs considèrent que le marché du travail espagnol présente l'un des niveaux de flexibilité contractuelle les plus élevés d'Europe (Banyuls et al., 2009). Cette proportion élevée de CDD est sans doute le facteur principal du fort accroissement de parcours professionnels non linéaires en Espagne, marqués par une forte variabilité et une grande discontinuité.

Dans ce contexte, l'article analysera le rôle que peut jouer la formation dans la stabilisation des parcours professionnels (voire dans le développement de parcours ascendants). Dans cette perspective, nous analyserons les effets de la formation en prenant appui sur l'approche par les capacités (Sen, 1999, 2002, 2006). Ceci implique de mettre davantage l'accent sur les modes et possibilités d'utilisation des ressources formatives, plutôt que sur le volume ou les caractéristiques de ces ressources. En ce sens, la formation est considérée comme un élément de renforcement des capacités (Salais et Villeneuve, 2004), dont l'objectif est d'accroître les opportunités d'emploi. Nous ne traiterons pas des effets de la formation sur l'insertion professionnelle mais sur les situations d'emploi et de contrat, après la période d'insertion professionnelle.

L'article montrera, dans un premier temps, l'utilité de l'approche par les capacités d'Amartya Sen pour analyser le rôle de la formation dans le déroulement des carrières professionnelles. Nous présenterons ensuite quelques éléments relatifs au contexte économique catalan et espagnol, avant de dresser un tableau sur l'organisation du marché du travail, et de présenter le cadre institutionnel de la formation en vigueur en Espagne. Nous présenterons ensuite la base de données utilisée et les résultats des différentes exploitations statistiques réalisées. Enfin, l'article se conclura par quelques remarques synthétiques sur ces différents éléments théoriques et empiriques.

\section{L'IMPACT DE LA FORMATION SUR LES PARCOURS DES INDIVIDUS SUR LE MARCHÉ DU TRAVAIL}

Sen (1993) préconise d'employer la notion de capacité par opposition à celle de ressources, comme base informationnelle de jugement des politiques publiques. Selon cette approche, et en considérant également l'influence de facteurs personnels et institutionnels (Robeyns, 2005), on peut envisager les parcours professionnels comme des ensembles d'accomplissements qui résultent de dotations différentes en capacités (Bonvin et Farvaque, 2008). Dans un contexte comme celui de l'Espagne, marqué par des incertitudes croissantes en matière de carrières professionnelles, l'approche par les capacités offre un cadre analytique permettant d'étudier dans quelle mesure les ressources fournies par les politiques sociales permettent aux travailleurs d'avoir une prise sur le déroulement de leur parcours (Verd, Vero et López Andreu, 2009).

Notre analyse, qui s'inscrit dans le cadre du projet de recherche CAPRIGHT${ }^{2}$, nous permet d'examiner

\footnotetext{
${ }^{2}$ Le projet CAPRIGHT (Resources, rights and capabilities: in search of social foundations for Europe) est coordonné par Robert Salais et financé par le VIe Programme Cadre de la Commission européenne (contrat CIT4-CT-2006-028549).
} 
si les ressources formatives modifient ou influencent la situation des travailleurs. Il faut donc imaginer, du point de vue analytique, la situation antérieure à l'utilisation des ressources (dérivant d'un ensemble préalable d'options, ressources et facteurs de conver$\left.\operatorname{sion}^{3}\right)$ qui, ici, se référera à la situation professionnelle (catégorie sociale) et occupationnelle (travail temporaire et chômage). À partir de cette situation initiale, on examinera si l'accès à certaines ressources formatives déterminées (qui seront décrites au paragraphe suivant) peut être corrélé à un changement de situation professionnelle et/ou occupationnelle. On examinera, du point de vue opérationnel, si la situation en termes de catégorie socioprofessionnelle, de contrat et de chômage au moment « $\mathrm{t}$ » (en l'espèce, l'année 2001) est modifiée ${ }^{4}$ en « $\mathrm{t}+\mathrm{n} »(2006)$ et si, dans le même laps de temps, l'intéressé a eu accès à une ressource formative. Notons que Sen (1999, Chap. 2) souligne que les capacités ne peuvent être correctement identifiées que si l'on a préalablement étudié les différents modèles et possibilités d'utilisation des ressources auxquelles les personnes ont potentiellement accès. À cet égard, l'auteur pointe la différence entre l'accès (ou droit) formel à une ressource et l'accès (ou droit) réel. Cet aspect du modèle de Sen est important dans la mesure où l'accès à la formation de la population ne se produit pas dans des conditions d'égalité absolue. Ainsi, dans l'analyse, nous avons tenu compte des caractéristiques sociales des travailleurs, non seulement de leurs caractéristiques individuelles, mais en prenant également comme variable indépendante le type de parcours professionnel réalisé. Ainsi nous

\footnotetext{
${ }^{3}$ Un facteur de conversion est une caractéristique individuelle ou de l'environnement de l'individu qui intervient entre les ressources qu'un individu pourrait potentiellement utiliser et celles que finalement il peut utiliser de manière effective. Par exemple, le droit individuel à la formation dans l'entreprise (qui existe aussi bien en France qu'en Espagne) résulte de la politique de formation de l'entreprise elle-même, de l'adéquation de la formation au profil du travailleur ou des disponibilités temporelles. Ces facteurs (entre autres) font que le droit formel se traduit en une possibilité réelle (capacité) et finalement en un accomplissement. Robeyns (2003) classifie les facteurs de conversion en trois groupes : (1) les facteurs sociaux (normes sociales, préjugés, facteurs culturels et religieux...), (2) les facteurs personnels (talents, compétences, connaissances) et (3) les facteurs environnementaux (lieu de résidence, infrastructures...).

${ }^{4}$ Dans le modèle de Sen, cette situation (accomplissement) en $\mathrm{t}+\mathrm{n}$ doit résulter du libre choix des individus. Nous considérons, dans notre analyse, que les promotions professionnelles et les changements de contrat et de situation d'emploi observés résultent de ce type de choix (parmi l'ensemble des options possibles, c'està-dire des capacités).
}

avons corrélé les usages et les effets de la formation avec les caractéristiques des personnes et celles de leur parcours professionnel.

\section{DES PARCOURS PROFESSIONNELS QUI S'INSCRIVENT DANS UN MARCHÉ DU TRAVAIL FLUCTUANT}

Dans cette partie, nous présenterons brièvement les évolutions du marché du travail en Espagne et en Catalogne, ainsi que le cadre institutionnel de la formation pour les salariés et les demandeurs d'emploi ; cela nous permettra d'évaluer l'influence de la formation sur le déroulement des parcours professionnels de la population étudiée.

\section{L'évolution de l'emploi en Espagne et en Catalogne}

Comme mentionné dans la partie précédente, notre analyse se centrera sur la période 2001-2006, caractérisée en Espagne par de forts taux de croissance économique et d'emploi et par de faibles taux de chômage. L'examen de cette période est particulièrement pertinent pour notre étude ; en effet, il existe probablement un certain effet de conjoncture d'amélioration généralisée de la situation sur le marché du travail, tant du point de vue de la mobilité interne (promotion) que de la mobilité externe (passage du chômage à l'emploi ou d'un CDD à un CDI). De même, cette situation économique favorable peut être corrélée avec le faible taux d'accès à la formation non formelle-notamment la formation occupationnelle ${ }^{5}$ - qui, bien qu'elle soit

\footnotetext{
${ }^{5} \mathrm{La}$ formation occupationnelle est une catégorie éminemment espagnole. Elle s'inscrit dans le cadre de la formation non formelle (Dautrey, 2007, p. 107). "En 1980, la Loi fondamentale sur l'emploi établira une distinction entre occupationnel et professionnel et qualifiera d'occupationnel un autre type de savoir professionnel ne s'appuyant ni sur la réglementation ni sur les pratiques d'enseignement initial'. A l'origine, la formation occupationnelle vise à l'insertion et la réinsertion dans le morde du travail des travailleurs au chomage ou des jeunes". http://www.aeht.lu/code/ educatio/espagn6.htm). "Durant les années 60, la formation occupationnelle cherchera à répondre aux besoins de main d'œuvre qualifiée engendrés par la croissance (...) Dès lors, la formation occupationnelle est liée aux qualifications théoriques et pratiques nécessaires à l'exercice d'une activité professionnelle et orientées vers l'apprentissage des tâches propres à cet exercice" (Dautrey, 2007 , p. 107). En français, cette notion pourrait s'apparenter à “formation de retour à l'emploi" ou "d'adaptation à l'emploi".
} 
destinée aussi bien aux travailleurs qu'aux chômeurs, est principalement suivie par ces derniers.

Soulignons que la forte flexibilité des recrutements, qui caractérise le marché du travail espagnol, amplifie les effets sur l'emploi des périodes de croissance et de décroissance économiques. Segura et al. (1990) constataient déjà, au début des années 90, que les facilités d'embauche en contrat à durée déterminée avaient accru la sensibilité cyclique de l'emploi, dans le sens où, en périodes de croissance économique, celle-ci augmenterait fortement, alors que la destruction de l'emploi serait beaucoup plus importante en périodes de stagnation économique. Ces créations et destructions d'emplois se concentrent dans des segments très précis. Plusieurs auteurs (Toharia et Malo, 2000, parmi les plus connus) considèrent que le marché du travail espagnol se caractérise par une forte segmentation entre emplois à durée indéterminée et emplois temporaires, ce qu'il a été convenu d'appeler la flexibilité à la marge. Ainsi, l'évolution économique du pays affecte principalement - positivement ou négativement - (et fortement) le segment des contrats temporaires.

Cette croissance de l'emploi au cours de la période analysée n'a pas été homogène dans tous les secteurs ou pour les différents types de professions. En Espagne, le poids de secteurs forts consommateurs de force de travail et à faible productivité (construction et services aux emplois à faible qualification) a engendré une forte croissance des emplois faiblement qualifiés. Cependant, le taux d'emploi a également augmenté pour les catégories professionnelles les plus qualifiées. Ainsi, Caprile et Potrony (2010) analysent l'évolution de l'emploi dans la période 1996-2007 à partir des résultats de l'Enquête sur la Population Active. Ils observent que le changement dans la structure de l'emploi est important et que la création d'emplois s'est concentrée sur les groupes extrêmes en termes de qualification. Cette dynamique est corroborée par les rapports du Conseil Économique et Social (2009), qui indiquent que cette période a été marquée par la création d'emplois au sein des groupes les plus et les moins qualifiés du marché du travail.

En Catalogne, au cours de cette période, la situation a été identique à celle du reste de l'Espagne. Comme le notent Martín (2007) et Caprile et al. (2008), la croissance économique qui a débuté dans la seconde moitié des années 90, s'est également accompagnée, en Catalogne, d'une forte augmentation du taux d'activité et d'emploi. Au niveau de la structure des emplois, Caprile et Potrony (2010) montrent, à l'aide des Enquêtes sur les Forces de Travail (LFS) d'Eurostat, que pourtant seulement $24 \%$ de la population est " cadre ou profession libérale "; cela correspond à la situation de l'Espagne, mais reste très inférieur aux $31 \%$ observés dans l'UE-15; et ce bien que le taux de diplômés de l'enseignement supérieur soit, en Catalogne, similaire à celui des autres pays et régions de l'UE-15 (Homs et al., 2006).

La situation actuelle de crise économique a bouleversé l'évolution de l'emploi, en générant une forte destruction d'emplois (notamment des CDD). Comme l'ont souligné Caprile et Potrony (2010), dans la période 2007-2009, le taux d'emploi a baissé de plus de 7 points en Catalogne, tandis que, sur la même période, le taux de chômage augmentait de 10 points (pour atteindre $16 \%$ ). La destruction d'emplois ayant touché tout particulièrement les travailleurs en CDD, le taux d'emploi temporaire a diminué de 6 points en Catalogne, et d'autant en Espagne. Néanmoins, il se situe à $18 \%$ dans le premier cas, contre $25 \%$ dans le second.

\section{Le cadre institutionnel de la formation des salariés et des demandeurs d'emploi}

En Espagne, au cours de la période considérée, la formation formelle s'organise sur la base de la Loi d'Aménagement du Système Éducatif de 1990 (LOGSE). Cette loi préconise d'instaurer une période de scolarité obligatoire jusqu'à 16 ans, puis de distinguer entre une période de deux ans d'Éducation Secondaire non obligatoire (pour ceux qui souhaitent suivre des études universitaires) et les Cycles de Formation de Niveau Moyen (pour ceux qui souhaitent bénéficier d'une formation professionnelle). À 18 ans, il est possible d'accéder aux Cycles de Formation de Niveau Supérieur ou à l'Université ${ }^{6}$. Cet ensemble de formations (professionnelles ou non) est dénommé

${ }^{6}$ En 2002 le Parti Populaire a apporté une modification à ce modèle en promulguant la Loi Organique de Qualité de l'Éducation, qui visait à introduire de facto, dès l'âge de 14 ans, une séparation entre la filière " académique » et la filière professionnelle (Merino, 2006, p. 64). En 2004, le changement de gouvernement a 


\section{Encadré 1 \\ La base de données utilisée}

Nous utilisons, dans notre analyse', les données du Panel sur les Inégalités (PaD) de la Fondation Jaume Bofill. Ces données fournissent des informations sur un échantillon d'individus de plus de 16 ans, issus de 2000 ménages Catalans. Le PaD inclut deux types de questionnaire, l'un sur le ménage et l'autre individuel. C'est ce dernier que nous avons utilisé. Nous avons donc travaillé avec un échantillon initial, sur la première vague, de 4880 personnes, avec une marge d'erreur de $+/-2,78$. À partir de cet échantillon initial, dans chacune des vagues, il y a une attrition de l'échantillon. II s'agit de personnes pour lesquelles il est impossible de recueillir des informations, soit parce qu'elles sont absentes de leur domicile, soient parce qu'elles ont déménagé sans laisser de trace de leur nouvelle adresse, etc. L'analyse se concentre sur les personnes âgées de 25 à 65 ans et en activité au cours de la période 200 1-2006. Nous avons écarté les personnes de moins de 25 ans, afin de réduire les distorsions dues à la prise en compte, dans l'analyse, des jeunes qui concilient études et emploi ou qui connaissent des situations très marquées dans l'entrée dans la vie active linactivité dans les toutes premières années sur le marché du travail). Dans ces cas, notre objectif - qui consiste à analyser l'impact de la formation suivie sur la mobilité interne et externe - subit une distorsion due au faible temps de présence sur le marché du travail (dans de nombreux cas, moins de 5 ans d'activité) et aux dynamiques résultant des processus diffus d'insertion sur le marché du travail, que l'on rencontre en Espagne.

Il convient de signaler que, dans l'échantillon finalement utilisé, le pourcentage de personnes ayant suivi une formation est très faible (cf. tableau 3), notamment s'agissant de la formation occupationnelle. Cette situation est relativement logique, vu le faible taux de chômage au cours de cette période. Le nombre de personnes ayant suivi une formation en entreprise est également faible, et seule la formation non formelle, résultant d'une initiative individuelle, est notable.

1 Ce travail n'aurait pas été possible sans l'aide inestimable de Àngels Llorens, de la Fondation Bofill, qui a mis à notre disposition toutes ses connaissances, tant en ce qui concerne l'accès, l'organisation et le traitement des données que les problèmes rencontrés au cours du travail dans le traitement des données.

formation formelle, dans la mesure où elles sont dispensées dans le cadre du système éducatif « formalisé ».

S'agissant de la formation professionnelle (FP), soulignons que la LOGSE établit une distinction entre la formation aboutissant à l'obtention du diplôme de technicien (Cycles de Formation de Niveau Moyen qui - comme nous venons de l'indiquer - sont intégrés dans l'enseignement secondaire post-obligatoire) et celle permettant d'obtenir le titre de technicien supérieur (Cycles de Formation de Niveau Supérieur), intégré dans l'enseignement supérieur non universitaire.

D'autre part, en Espagne il existe diverses initiatives de formation, en dehors du système de formation professionnelle que nous venons de décrire, générale-

empêché la mise en œuvre de cette loi. Est alors entrée en vigueur la réforme de la LOGSE de 2006, très similaire à la loi de 1990. ment regroupées sous l'appellation de formation non formelle. Dans ce contexte, on distingue généralement entre formation continue (désignant les actions de formation des entreprises et les congés individuels de formation) et formation occupationnelle (ne relevant pas de l'initiative des entreprises ; elle est destinée aux demandeurs d'emploi, mais est également ouverte aux travailleurs). Ces deux formations sont formellement structurées et intégrées dans le sous-système de « formation professionnelle pour l'emploi », englobant également la formation en alternance, alternant des périodes de travail en entreprise et des périodes de formation.

Notons enfin le poids que représente, en Espagne, l'ensemble des formations complémentaires, qui font partie du système non formel (cours de langues, bureautique, informatique, etc.), qui contribuent à accroître les compétences des travailleurs et qui relèvent de l'initiative individuelle. 


\section{LES EFFETS DE LA FORMATION DIFFÈRENT SELON LE PARCOURS PROFESSIONNEL}

\section{La formation ne favorise pas toujours la mobilité interne ou externe}

Dans l'échantillon, le faible nombre d'individus ayant suivi une formation permet difficilement de parvenir à des résultats définitifs ; cependant, nous avons réalisé, sur la base des données du $\mathrm{PaD}$, une première analyse fondée sur des régressions logistiques multinomiales ( $c f$. Tableau 1). Cette analyse vise à identifier le rapport entre le suivi d'un type donné de formation et les trois types de changement professionnel que les données permettaient d'envisager : a) changement de catégorie professionnelle (promotion ou déclassement) ${ }^{7}$, b) changement de contrat (passage d'un CDD à un CDI, ou inversement) etc.) changement de situation d'emploi (passage de chômeur à employé, ou inversement). Cette analyse ne permet certes pas d'induire des relations strictes de cause à effet - vu qu'elle compare les situations des travailleurs entre la première vague de l'enquête (2001) et la dernière vague disponible à la date de l'analyse (2006) ; néanmoins, il devrait être possible de repérer la coïncidence, au cours de la période, d'expériences de formation non formelles ou d'augmentation du niveau d'études formelles, avec la mobilité, positive ou négative, que nous venons d'évoquer. Rappelons que, dans notre approche, la formation est considérée comme une ressource qui devrait théoriquement élargir le champ réel des possibilités d'emploi (capacités) des travailleurs. En ce sens, du point de vue des capacités, le système institutionnel de formation agirait sur les facteurs de conversion des individus, en leur fournissant aussi bien une certification que des savoirs qui devraient théoriquement améliorer leur situation professionnelle dans la période $« \mathrm{t}+\mathrm{n} »$.

\footnotetext{
${ }^{7}$ Nous avons appréhendé la mobilité interne sur la base des catégories suivantes : ouvrier non qualifié de l'industrie et des services ; ouvrier qualifié de l'industrie et des services ; administratifs et autres emplois non manuels ; technicien intermédiaire ; technicien supérieur et cadre. Sur cette base, nous avons calculé si, au cours de la période, l'intéressé est resté dans sa catégorie professionnelle ou en a changé dans le sens ascendant ou descendant.
}

Nous évaluerions donc, indirectement, l'efficacité de ce système institutionnel de formation ${ }^{8}$.

Dans les modèles de régression, la formation occupationnelle (fondamentalement destinée aux demandeurs d'emploi), la formation continue (à l'initiative des entreprises), la formation relevant de l'initiative individuelle et l'augmentation des études formelles ont été considérées comme des variables explicatives. En outre, nous avons introduit un ensemble de variables considérées comme des facteurs structurants du marché du travail, tels que le sexe, l'âge, le niveau d'études, la catégorie professionnelle, le secteur d'activité et la taille de l'établissement. Ces variables visent à préciser et contrôler les effets propres de la formation.

Les résultats issus de cette première analyse n'ont pas fait apparaître une influence décisive de la formation (cf. tableau 1). En effet, aussi bien dans le cas de la mobilité interne ascendante (promotion professionnelle) que dans celui de l'amélioration du contrat (passage d'un CDD à un CDI) ou de l'amélioration de la situation d'emploi (passage de chômeur à travailleur), les résultats sont contradictoires : recevoir une formation est, pour certains types de formation, autant corrélé avec une évolution positive que négative (changement négatif de catégorie, passage d'un CDI à un CDD ou passage de l'état d'emploi à celui de chômage). En résumé, à l'issue de cette première analyse, il est impossible de déduire un effet causal de la formation - formelle ou non - sur les changements de catégorie, de situation contractuelle ou de situation professionnelle. Les régressions réalisées montrent cependant des effets significatifs d'autres variables, telles que le secteur ou la catégorie professionnelle au début de la période (probablement liés à la prospérité économique de la période analysée), ou les effets de la taille de l'établissement (en cas de changement de contrat) ou du sexe (en cas de changement de situation professionnelle).

Ainsi, les variables de formation ne sont pas significativement corrélées avec la situation professionnelle. En nous référant à l'ensemble de la population, nous avons procédé à une analyse plus fine, présentée

\footnotetext{
${ }^{8}$ Soulignons que, pour Sen (1999), une politique publique adaptée est une politique qui combine la garantie des droits ou des biens et services avec la mise en place des facteurs de conversion adéquats.
} 
Tableau 1

Analyse de regresión logistique

\begin{tabular}{|c|c|c|c|c|c|c|}
\hline \multirow[t]{2}{*}{$\begin{array}{c}\text { Variables } \\
\text { indépendantes }\end{array}$} & \multicolumn{2}{|c|}{$\begin{array}{c}\text { Modelo } 1 \\
\text { (catégorie professionnelle) }\end{array}$} & \multicolumn{2}{|c|}{$\begin{array}{c}\text { Modelo } 2 \\
\text { (contrat) }\end{array}$} & \multicolumn{2}{|c|}{$\begin{array}{l}\text { Modelo } 3 \\
\text { (chômage) }\end{array}$} \\
\hline & Promotion & $\begin{array}{c}\text { Régression } \\
\text { de catégorie } \\
\text { professionnelle }\end{array}$ & $\begin{array}{c}\text { Travail } \\
\text { temporaire } \\
\text { à CDI }\end{array}$ & $\begin{array}{l}\text { CDI à travail } \\
\text { temporaire }\end{array}$ & $\begin{array}{l}\text { Chômeur } \\
\text { à employé }\end{array}$ & $\begin{array}{l}\text { Employé } \\
\text { à chômeur }\end{array}$ \\
\hline$\hat{A}$ Age & ns & 0,03 & $-0,04$ & $-0,08$ & $-0,07$ & ns \\
\hline \multicolumn{7}{|l|}{ Sexe } \\
\hline Homme & Réf. & Réf. & Réf. & Réf. & Réf. & Réf. \\
\hline Femme & $\mathrm{ns}$ & ns & ns & ns & ns & 1,23 \\
\hline \multicolumn{7}{|l|}{ Niveau d'études } \\
\hline Enseignement secondaire & Réf. & Réf. & Réf. & Réf. & Réf. & Réf. \\
\hline Enseignement primaire & ns & $-1,10$ & ns & ns & ns & $\mathrm{ns}$ \\
\hline CAP-BEP & ns & ns & ns & ns & ns & ns \\
\hline BTS-DUT & ns & ns & ns & ns & ns & ns \\
\hline Licence & ns & ns & ns & ns & ns & ns \\
\hline Maîtrise & ns & 1,14 & ns & $-1,10$ & ns & ns \\
\hline $\begin{array}{r}\text { Master (ex dea, dess), } \\
\text { doctorat }\end{array}$ & ns & 1,36 & ns & & ns & ns \\
\hline \multicolumn{7}{|l|}{ Catégorie socioprofessionnelle } \\
\hline Travailleurs non qualifiés & Réf. & Réf. & Réf. & Réf. & Réf. & Réf. \\
\hline Directeur/Gérant & $2,82^{*}$ & ns & ns & ns & ns & ns \\
\hline Techniciens supérieurs & 3,21 & $-4,06$ & ns & ns & $-2,65$ & $-3,15$ \\
\hline Techniciens moyens & 2,96 & $-1,95$ & ns & ns & $-1,49$ & ns \\
\hline $\begin{array}{r}\text { Administratifs et autres non } \\
\text { manuels }\end{array}$ & 2,40 & $-0,70$ & ns & $-1,26$ & ns & $-1,57$ \\
\hline Travailleurs qualifiés & 1,59 & $-1,57$ & ns & ns & $-1,07$ & $-1,63$ \\
\hline \multicolumn{7}{|l|}{ Secteur d'activité } \\
\hline Services & Réf. & Réf. & Réf. & Réf. & Réf. & Réf. \\
\hline Agriculture & $\mathrm{ns}$ & ns & ns & ns & ns & ns \\
\hline Industrie & ns & ns & ns & ns & ns & 1,44 \\
\hline Bâtiment & ns & ns & 1,24 & ns & ns & ns \\
\hline \multicolumn{7}{|l|}{ Effectif de l'entreprise } \\
\hline 1 à 10 & Réf. & Réf. & Réf. & Réf. & Réf. & Réf. \\
\hline 11 à 30 & ns & ns & ns & ns & ns & ns \\
\hline 31 à 50 & ns & ns & ns & ns & ns & ns \\
\hline Plus de 50 & ns & ns & $-1,09$ & ns & ns & ns \\
\hline $\begin{array}{l}\text { Formation non formelle } \\
\text { individuelle }\end{array}$ & 1,96 & 1,33 & ns & 1,74 & ns & 3,03 \\
\hline $\begin{array}{l}\text { Formation non formelle } \\
\text { occupationnelle }\end{array}$ & ns & ns & ns & ns & ns & ns \\
\hline
\end{tabular}




\begin{tabular}{|c|c|c|c|c|c|c|}
\hline \multirow[t]{2}{*}{$\begin{array}{c}\text { Variables } \\
\text { indépendantes }\end{array}$} & \multicolumn{2}{|c|}{$\begin{array}{l}\text { Modelo } 1 \\
\text { (catégorie professionnelle) }\end{array}$} & \multicolumn{2}{|c|}{$\begin{array}{c}\text { Modelo } 2 \\
\text { (contrat) }\end{array}$} & \multicolumn{2}{|c|}{$\begin{array}{l}\text { Modelo } 3 \\
\text { (chômage) }\end{array}$} \\
\hline & Promotion & $\begin{array}{c}\text { Régression } \\
\text { de catégorie } \\
\text { professionnelle }\end{array}$ & $\begin{array}{l}\text { Travail } \\
\text { temporaire } \\
\text { à CDI }\end{array}$ & $\begin{array}{l}\text { CDI à travail } \\
\text { temporaire }\end{array}$ & $\begin{array}{c}\text { Chômeur } \\
\text { à employé }\end{array}$ & $\begin{array}{l}\text { Employé } \\
\text { à chômeur }\end{array}$ \\
\hline $\begin{array}{l}\text { Augmentation du niveau } \\
\text { d'études formelles }\end{array}$ & ns & ns & ns & ns & ns & ns \\
\hline Constante & $-2,05$ & ns & ns & ns & ns & $-4,00$ \\
\hline \multicolumn{7}{|l|}{ Statistiques } \\
\hline R2 (Nagelkerke) & \multicolumn{2}{|c|}{0,366} & \multicolumn{2}{|c|}{0,209} & \multicolumn{2}{|c|}{0,243} \\
\hline Effectif (N) & \multicolumn{2}{|r|}{748} & \multicolumn{2}{|c|}{772} & \multicolumn{2}{|c|}{937} \\
\hline
\end{tabular}

Source : Auteur, sur la base du panel des inégalités (2001-2006). Fundació Jaume Bofill.

NB. : les chiffres sont significatifs au seuil de $5 \%$.

Note de lecture : Le changement positif de catégorie professionnelle (promotion) est significativement corrélé avec la catégorie professionnelle : être dirigeant ou gérant (2,82\% fois, plus que la référence "Travailleurs non qualifiés").

ci-après, en repérant différentes logiques dans les parcours professionnels.

\section{Les inégalités en matière de travail temporaire, de chômage et $d$ 'inactivité génèrent des parcours instables}

Il s'agit ici d'apporter à l'analyse un contenu sociologique plus marqué et, en même temps, d'identifier certaines règles plus évidentes d'interprétation. À cet effet, nous exposons ci-après les différents profils de parcours, obtenus empiriquement par une classification automatique (cluster analysis) appliquée aux mêmes données du PaD. Cette identification de différents groupes, sur la base des diverses transitions et situations professionnelles vécues, permet d'inférer le « modèle professionnel » dans lequel s'inscrit la formation. Comme nous l'avons vu dans la partie précédente, il n'existe pas de "règle universelle » permettant de corréler formation et mobilité ; c'est pourquoi il est nécessaire de « segmenter » l'analyse, en se référant aux différents parcours que l'on observe sur le marché du travail espagnol.

Soulignons le lien qui existe entre cette identification de différents types de parcours et les réflexions de Sen (1999) concernant la nécessité de considérer les circonstances et les contextes dans lesquels les ressources (en l'occurrence, les ressources formatives) sont « offertes » aux personnes. L'identification de différents types de parcours permet non seulement de déterminer divers contextes de vie et de travail dans lesquels les ressources formatives sont potentiellement utilisées, mais aussi d'inscrire dans une logique déterminée l'utilisation de cette formation. C'est ainsi que le concept de capacité à l'emploi (l'ensemble réel des opportunités d'emploi) prend son sens spécifique dans l'ensemble des possibilités d'emploi, passées et futures, d'un type donné de parcours. Un certain type de formation peut apporter, pour un type de parcours déterminé, une amélioration ou un maintien des possibilités d'emploi, alors que, pour un autre type de parcours, le même genre de formation n'aura pas d'effet par rapport aux possibilités antérieures à la formation.

Les variables utilisées pour l'analyse de classification regroupent, pour les cinq vagues consécutives (2001-2002 à 2006), les informations suivantes : (a) fréquences de travail temporaire, d'inactivité et de chômage au cours de la période, dont la valeur minimale est « 0 » et la valeur maximale « $5 »$; (b) transitions entre chômage et emploi et entre CDD et CDI, dans la première et dernière vague, dont la valeur minimale est $«-1 »$ (dans les transitions négatives) et la valeur maximale « $1 »$ (dans les transitions positives) ; et (c) l'augmentation du niveau d'études formelles, entre la première et la dernière vague (dont la valeur minimale est $« 0$ et la valeur maximale « $1 »)$ et la réalisation de formation non formelle (avec valeur minimale « 0 » et valeur maximale « $3 »)$. Comme dans l'analyse de 
Tableau 2

Type de parcours et moyenne des variables considérées. PaD (2001-2006)

\begin{tabular}{|c|c|c|c|c|c|c|c|c|}
\hline Type de parcours & & 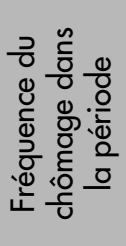 & 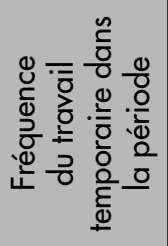 & 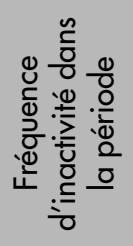 & 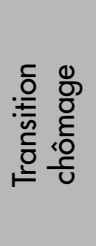 & 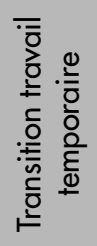 & 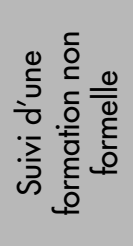 & 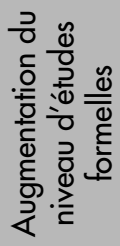 \\
\hline \multirow[t]{2}{*}{ 1-Linéaire } & moyenne & 0,1 & 0,0 & 0,1 & 0,0 & 0,0 & 0,1 & 0,2 \\
\hline & $N$ & 359 & 359 & 359 & 359 & 359 & 359 & 359 \\
\hline \multirow[t]{2}{*}{ 2-Professionnel } & moyenne & 0,1 & 0,0 & 0,1 & 0,0 & 0,0 & 1,4 & 0,0 \\
\hline & $N$ & 186 & 186 & 186 & 186 & 186 & 186 & 186 \\
\hline \multirow[t]{2}{*}{ 3-Féminin discontinu } & moyenne & 0,3 & 0,3 & 3,1 & 0,1 & 0,0 & 0,2 & 0,0 \\
\hline & $\mathrm{N}$ & 70 & 70 & 70 & 70 & 70 & 70 & 70 \\
\hline \multirow[t]{2}{*}{ 4-Précarité } & moyenne & 0,6 & 1,7 & 0,4 & 0,1 & 0,0 & 0,5 & 0,1 \\
\hline & $N$ & 189 & 189 & 189 & 189 & 189 & 189 & 189 \\
\hline \multirow{2}{*}{$\begin{array}{l}\text { 5-Travail temporaire } \\
\text { chronique }\end{array}$} & moyenne & 0,1 & 4,1 & 0,1 & 0,1 & $-0,4$ & 0,7 & 0,3 \\
\hline & $N$ & 80 & 80 & 80 & 80 & 80 & 80 & 80 \\
\hline \multirow[t]{2}{*}{ Total } & moyenne & 0,2 & 0,8 & 0,4 & 0,0 & 0,0 & 0,5 & 0,1 \\
\hline & $N$ & 884 & 884 & 884 & 884 & 884 & 884 & 884 \\
\hline
\end{tabular}

Source : Établi par les auteurs sur la base du Panel sur les Inégalités (2001-2006). F. Bofill.

Exemple de lecture : Pour le parcours linéraire, on peut lire que, entre 2001 et 2006, les individus ont été 0,1 fois au chômage en moyenne, n'ont pas connu de période de travail temporaire, et ont été inactifs 0,1 fois en moyenne ; en comparaison avec la situation uniquement au début et à la fin de la période analysée, ils n'ont connu aucun changement du chômage à l'emploi (ni le contraire), ni de contrat temporaire à stable (ni le contraire); la moitié ont suivi 0,1 fois une formation non formelle pendant les trois années pour lesquelles nous disposons de cette variable, et ils ont augmenté leur niveau d'études formelles de 0,2 sur 1 (20\% des cas ont augmenté leur niveau d'études formelles entre la première et la dernière vague).

régression évoquée au chapitre précédent, il s'agit de personnes âgées de 25 à 65 ans qui ont parfois été actives au cours de la période 2001-2006. Au total, 884 personnes présentent des valeurs renseignées pour toutes les variables considérées. Nous avons finalement retenu cinq types de parcours. Le tableau 2 présente ces types de parcours, le nombre de personnes concernées et la valeur moyenne pour chaque type de parcours de chacune des variables.

Après avoir construit la typologie des parcours, nous la décrirons sommairement sur la base des : (a) variables introduites dans l'analyse (variables actives) ; (b) variables relatives au salaire et au parcours professionnel, obtenues par Caprile et Potrony $(2007)^{9}$, et

\footnotetext{
9 Nous remercions Maria Caprile et Jordi Potrony pour avoir mis à notre disposition les variables de parcours et de salaire que nous avons utilisées dans la caractérisation des clusters (Caprile et Potrony, 2007).
}

(c) variables de caractéristiques personnelles et du marché du travail:

Le type 1 ou Parcours Linéaire (41\% des cas) se caractérise par le faible poids des transitions, avec un salaire se situant dans la moyenne, peu d'expérience du chômage et, lorsque c'est le cas, de faible durée. Il s'agit d'un groupe majoritairement constitué d'hommes, ayant atteint un niveau d'études de base ou secondaires, d'âge moyen (41 ans et plus), et majoritairement composé d'ouvriers non qualifiés, d'agents administratifs et autres travailleurs non manuels.

Le type 2 ou Parcours Professionnel (21\% des cas) est marqué par une aussi faible présence des transitions qu'au sein du premier groupe, mais avec une forte présence d'études non formelles. Il dispose du salaire le plus élevé, a peu l'expérience du chômage et, lorsque c'est le cas, il s'agit d'un chômage de courte durée. Il est constitué aussi bien d'hommes que de femmes, 
avec des cycles de formation de niveau supérieur, des diplômes universitaires. Ils ont un âge moyen et ce sont majoritairement des techniciens intermédiaires, suivis des techniciens supérieurs et cadres.

Le type 3 ou Parcours discontinu féminin (8\% des cas) se caractérise et se définit par une forte présence de l'inactivité au cours des années analysées. Le salaire moyen est très bas, fortement marqué par le chômage et souvent de longue durée. Il est presque exclusivement constitué de femmes, avec un niveau d'études de base, mais aussi d'études secondaires. Ce groupe est constitué de personnes de 50 à 65 ans, mais aussi de moins de 30 ans.

Le type 4 ou Parcours de précarité (21\% des cas) se caractérise par une certaine présence du chômage au cours de la période analysée, ainsi que par la présence $\mathrm{du}$ travail temporaire. Le salaire est bas, avec un parcours relativement marqué par le chômage qui est, dans ce cas, d'une certaine durée. C'est un groupe majoritairement composé de femmes, avec un niveau d'études de base, mais parfois avec des diplômes universitaires. La tranche d'âge est globalement de 26 à 35 ans. Il s'agit majoritairement d'ouvriers non qualifiés mais aussi, dans une moindre mesure, d'ouvriers qualifiés.

Enfin, le type 5 ou Parcours de travail temporaire chronique ( $9 \%$ des cas) est marqué par le travail temporaire, qui devient quasiment la règle pour les travailleurs de ce groupe, au cours des années analysées. On trouve, dans ce profil, de la formation non formelle. Ce groupe enregistre la plus forte augmentation du niveau d'études formelles. Le salaire moyen est faible, avec une certaine présence du chômage - de durée moyenne - dans le parcours. Il s'agit d'un groupe constitué aussi bien d'hommes que de femmes, ayant suivi une formation professionnelle (FP) de niveau moyen et supérieur, relativement importante, mais possédant parfois aussi des diplômes universitaires. L'âge moyen se situe majoritairement entre 26 et 35 ans. La catégorie la plus représentée est celle des techniciens intermédiaires, suivis des ouvriers qualifiés.

\section{Parcours, transitions et formation}

Après avoir constitué les différents types de parcours, nous analysons, pour chacun d'entre eux, le lien entre la participation à la formation (formelle ou non) et les mobilités externes et internes qu'ils ont connues au cours de la période. À cet effet, nous avons élaboré les tableaux 3 et 4 , qui décrivent le type de transitions et de formations les plus fréquentes dans chaque type de parcours, mais qui n'ont aucun caractère explicatif. Il n'est pas pertinent d'effectuer une nouvelle régression avec la variable «type de parcours » (chacun des groupes obtenus) comme variable indépendante puisque ces groupes ont été partiellement construits sur la base de nos propres variables de formation. Les tableaux que nous présentons doivent donc être considérés comme un moyen d'introduire une logique sociologique et contextualisante permettant, d'une certaine façon, d'associer sur un mode descriptif chaque type de parcours à un certain type de transition et d'utilisation de la formation.

Tout d'abord, au vu des tableaux 3 et 4 , on observe l'inégale distribution des transitions et des types de formation entre les profils obtenus. Ceci n'est pas surprenant dans la mesure où ces variables ont été employées, entre autres ( $c f$. tableau 3), pour la constitution des types de parcours. Par ailleurs, le tableau 4 montre qu'un très faible pourcentage de l'échantillon a suivi une formation non formelle dans la période 2005-2006 (années pour lesquelles on dispose de ces données sur la formation). Le tableau 4 montre aussi que $7,6 \%$ de l'échantillon a augmenté son niveau de formation formelle, au cours de la période analysée (entre 2001 et 2006).

Si nous rapprochons les données du tableau 3 avec celles du tableau 4, certaines règles apparaissent, qui permettent de « segmenter » l'analyse du rôle de la formation, ainsi que nous l'avons énoncé au début de la sous-partie intitulée "Analyse de classification des parcours professionnels ». Si l'on considère les données relatives au changement de contrat, on observe que ce sont les Parcours de Précarité et de Travail Temporaire Chronique qui cumulent ce type de transition. Dans le premier profil, les transitions s'opèrent dans les deux sens, tandis que, dans le second, elles se font majoritairement vers le contrat à durée déterminée. Cela révèle l'instabilité contractuelle des deux types de profil. Les transitions vers le chômage sont pertinentes dans les parcours de type Linéaire et Précarité ; cela coïncide avec deux des trois groupes qui ont le plus augmenté leur niveau d'études formelles (le $3^{\mathrm{e}}$ est celui du Travail 
Tableau 3

Transitions professionnelles et occupationnelles par type de parcours

\begin{tabular}{|c|c|c|c|c|c|c|}
\hline & \multicolumn{5}{|c|}{ Type de parcours professionnels $(25-65)$} & \multirow[b]{2}{*}{ Total } \\
\hline & Linéaire & Professionnel & $\begin{array}{l}\text { Discontinu } \\
\text { féminin }\end{array}$ & Précarité & $\begin{array}{c}\text { Travail } \\
\text { temporaire } \\
\text { chronique }\end{array}$ & \\
\hline \multicolumn{7}{|c|}{$\begin{array}{l}\text { Changement de contrat (2001 à 2006) } \\
N=884\end{array}$} \\
\hline Pas de changement & $98,9 \%$ & $100,0 \%$ & $90,0 \%$ & $43,4 \%$ & $45,0 \%$ & $81,7 \%$ \\
\hline De CDI à CDD & $0,6 \%$ & $0,0 \%$ & $4,3 \%$ & $27,0 \%$ & $47,5 \%$ & $10,6 \%$ \\
\hline De CDD à CDI & $0,6 \%$ & $0,0 \%$ & $5,7 \%$ & $29,6 \%$ & $7,5 \%$ & $7,7 \%$ \\
\hline Total & $100,0 \%$ & $100,0 \%$ & $100,0 \%$ & $100,0 \%$ & $100,0 \%$ & $100,0 \%$ \\
\hline \multicolumn{7}{|c|}{$\begin{array}{l}\text { Changement de situation d'emploi (2001 à 2006) } \\
N=884\end{array}$} \\
\hline Pas de changement & $96,4 \%$ & $95,2 \%$ & $87,1 \%$ & $84,7 \%$ & $93,8 \%$ & $92,6 \%$ \\
\hline De travailleur à chômeur & $3,6 \%$ & $1,6 \%$ & $0,0 \%$ & $3,7 \%$ & $0,0 \%$ & $2,6 \%$ \\
\hline De chômeur à travailleur & $0,0 \%$ & $3,2 \%$ & $12,9 \%$ & $11,6 \%$ & $6,3 \%$ & $4,8 \%$ \\
\hline Total & $100,0 \%$ & $100,0 \%$ & $100,0 \%$ & $100,0 \%$ & $100,0 \%$ & $100,0 \%$ \\
\hline \multicolumn{7}{|c|}{$\begin{array}{l}\text { Changement de catégorie professionnelle ( } 2001 \text { a 2006) } \\
N=690\end{array}$} \\
\hline Pas de changement & $44,7 \%$ & $45,6 \%$ & $19,4 \%$ & $36,9 \%$ & $40,0 \%$ & $41,7 \%$ \\
\hline Recul & $32,0 \%$ & $23,4 \%$ & $16,7 \%$ & $26,2 \%$ & $21,5 \%$ & $27,2 \%$ \\
\hline Progression & $23,3 \%$ & $31,0 \%$ & $63,9 \%$ & $36,9 \%$ & $38,5 \%$ & $31,0 \%$ \\
\hline Total & $100,0 \%$ & $100,0 \%$ & $100,0 \%$ & $100,0 \%$ & $100,0 \%$ & $100,0 \%$ \\
\hline
\end{tabular}

Source : Établi par l'auteur sur la base du Panel sur les Inégalités (2001-2006). F. Bofill.

Exemple de lecture : Dans le parcours linéraire, $98,9 \%$ des individus n'ont pas connu de changement de contrat et 96,4\% n'ont pas changé de situation d'emploi. $44,7 \%$ n'ont pas changé de catégorie professionnelle.

Temporaire Chronique) et peut indiquer la tendance de certains groupes à mettre à profit la période de chômage pour atteindre ou accroître un certain niveau d'études formelles.

Par ailleurs, s'agissant des changements dans la situation d'emploi, les parcours de type Féminin Discontinu, de Travail Temporaire Chronique et de Précarité sont majoritairement passés de l'état de chômage à celui d'activité. Cette évolution positive peut s'expliquer par un certain effet de conjoncture comme nous l'avons déjà signalé dans la sous-partie «Quelques analyses sur l'évolution du marché du travail en Espagne et en Catalogne »-, mais elle peut aussi être le résultat positif du suivi d'une formation occupationnelle. Ce lien apparent entre formation occupationnelle et insertion pour certains groupes est corroboré par les études sur les effets de la forma- tion occupationnelle, réalisées par des auteurs tels que Mato (2002), Alonso Arellano (2005) et Mato et Cueto (2008), pour lesquels la participation à des stages de formation pour chômeurs ${ }^{10} \mathrm{n}$ 'apporte pas un bénifice à tous les participants.

Le parcours de type Travail Temporaire Chronique se caractérise, en outre, par l'élaboration de stratégies formatives à caractère individuel. Cette stratégie différenciée peut expliquer les résultats apparemment contradictoires relevés dans la régression logistique,

\footnotetext{
${ }^{10}$ Comme l'indiquent Demazière et Dubar (2004), les chômeurs ne constituent pas une population homogène. Leur situation ne s'explique pas seulement par des déterminants objectifs (tels que le niveau d'étude, l'âge, etc.), mais aussi par la projection, de la part du chômeur, des avenirs possibles, en liaison avec son passé (transaction biographique) et par la reconnaissance de la légitimité des aspirations et prétentions, de la part des acteurs institutionnels (transaction relationnelle).
} 
Tableau 4

Pourcentage des individus qui ont suivi formation formelle et non formelle par parcours

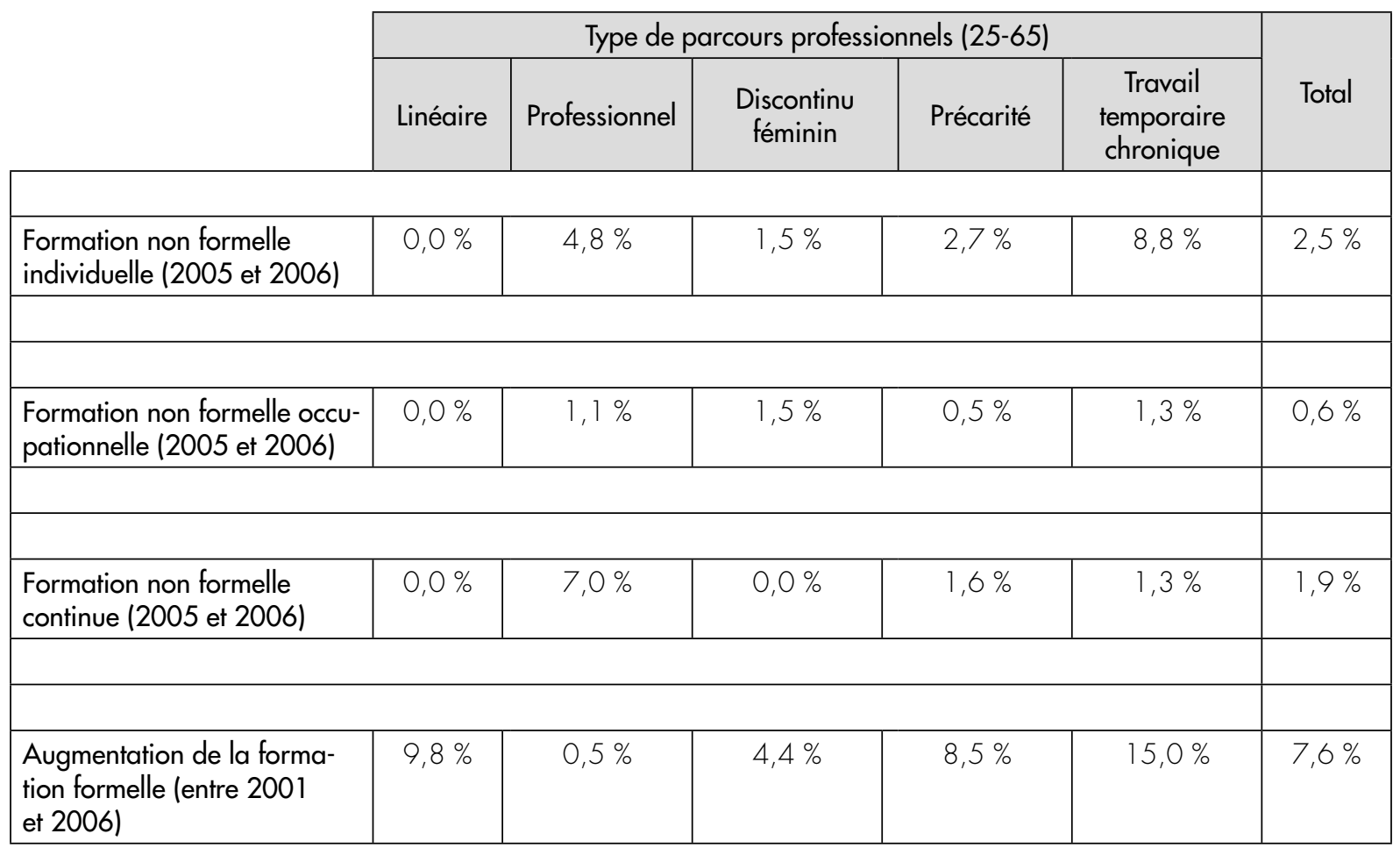

Source: réalisé par les auteurs sur la base du Panel sur les Inégalités (2001-2006). F. Bofill.

Exemple de lecture : dans le parcours linéraire, aucun individu n'a suivi de formation non formelle individuelle ni de formation non formelle occupationnelle, ni de formation formelle continue. $9,8 \%$ ont augmenté leur niveau de formation formelle.

s'agissant des effets de la formation non formelle individuelle. Le profil de Travail Temporaire Chronique est principalement composé de jeunes qui subissent une forte rotation d'emploi impliquant de fréquents changements de contrat et de situation professionnelle. Ainsi, le recours à la formation non formelle, qui caractérise le groupe, ne semble pas toujours déboucher sur une amélioration de leur situation professionnelle ou contractuelle.

Quant aux changements de catégorie professionnelle, le déclassement est particulièrement important dans le parcours de type Linéaire et, à un degré moindre, au sein du parcours de type Précarité. Les groupes qui bénéficient le plus d'un changement positif de catégorie sont ceux du parcours Travail Temporaire Chronique, de Précarité et Discontinu Féminin. Cela explique la corrélation négative, que l'on observe dans la régression logistique, entre le suivi d'une formation continue formelle (formation dans l'entreprise) et le changement positif de catégorie : en réalité, ce sont les personnes à parcours instable (qui n'ont pas nécessairement un faible niveau d'études) qui enregistrent un changement positif de catégorie, même si, par ailleurs, ce sont celles qui ont le moins bénéficié de la formation continue (d'où la corrélation négative entre changement positif de catégorie et participation à une formation continue).

S'agissant précisément de la formation continue, les éléments du tableau 4 permettent de nuancer quelque peu les recherches qui établissent une corrélation directe entre l'accession à la formation continue et les niveaux d'études. Certes, les profils les plus diplômés (parcours Professionnel) ont le plus accès à la formation dans les entreprises (Planas, 2005) ; cependant, ce rapport est très marqué par le degré de stabilité de la carrière professionnelle, étant donné que les profils à haut niveau de formation, mais à carrières professionnelles instables (tel que le profil Travail 
Temporaire Chronique), bénéficient de peu de formation en entreprise ${ }^{11}$.

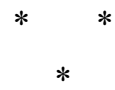

Nous présentons ci-après les conclusions de nos analyses. Soulignons que le faible nombre de cas qui composent notre échantillon, de même que le type de traitement des données - analyse de régression logistique, classification automatique et, en particulier, analyse des tableaux de contingence, auxquelles on ne peut attribuer un caractère explicatif - confèrent à ces conclusions un caractère exploratoire. Il s'agit plutôt de réflexions qui sont proposées comme des hypothèses restant à valider par des analyses ultérieures. D'autre part, bien que les données utilisées et les réflexions que nous exposons fassent référence au cas catalan, nous pensons que la réalité globale en Espagne n'est pas très éloignée de la situation que nous décrivons dans les paragraphes qui suivent.

\section{FORMATION ET PARCOURS PROFESSIONNELS EN CATALOGNE}

L'objectif qui consiste à analyser le rôle de la formation dans les changements professionnels et contractuels des travailleurs et dans leur capacité à faire face aux situations de risque potentiel ne peut pas être déconnecté, en Catalogne, ni dans l'ensemble de l'Espagne, du développement d'un marché du travail très flexible, notamment pour les jeunes et les femmes. En ce sens, l'interaction entre les caractéristiques du marché du travail et les stratégies individuelles génère des parcours professionnels marqués, pour une grande partie de la population, par une instabilité professionnelle qui perdure jusqu'à l'âge de 35 ou 40 ans $^{12}$. Ce processus de généralisation de la précarité (sous forme

\footnotetext{
${ }^{11}$ Les profils stables, mais à niveau de formation bas ou moyen, (profil de Parcours Linéaire) ne bénéficient pas non plus de la formation en entreprise.

${ }^{12}$ Les analyses de Virginia Hernanz (2003) de trois cohortes différentes (nées entre 1971-1974, 1966-1970 et 1961-1965) sur la fin du travail temporaire montrent que, à partir de 35 ans, le taux de travail temporaire chute à $17 \%$, pour les hommes, et jusqu'à $11 \%$ pour les femmes.
}

de chômage ou d'instabilité contractuelle) a déstructuré les parcours ouvriers traditionnels, en particulier chez les femmes. En ce sens, l'effet de la flexibilité se combine avec le processus d'intégration de la femme au sein du marché du travail et le développement (par rapport au passé) d'une économie espagnole particulièrement basée sur les services et le bâtiment.

En traitant les données analysées avec toutes les précautions requises, signalons d'abord le faible nombre des travailleurs qui suivent une formation non formelle. Globalement, cette situation peut s'expliquer par un effet de conjoncture, dans lequel de faibles taux de chômage entraînent, d'un point de vue individuel, un faible investissement dans la formation. Les chiffres semblent indiquer que la formation (formelle et non) se concentre sur certains types de profils, caractérisés par l'accumulation de diplômes.

Les données utilisées montrent également qu'analyser les effets de la formation indépendamment des différents types de parcours existants et de l'usage qu'ils font de la formation aboutit à des résultats peu significatifs, voire contradictoires. À cet égard, il s'avère fructueux d'identifier empiriquement différents types de parcours permettant de contextualiser et de conférer un sens sociologique aux utilisations des différents types de formation. Cette identification de divers types de parcours permet également de situer, dans une « carrière professionnelle » donnée, les types de formation suivis par les individus, ainsi que leurs effets. En ce sens, il est possible d'identifier ce que Sen appelle certains " modèles d'utilisation » des ressources formatives qui sont institutionnellement mis à la disposition de la population active.

\section{LA FORMATION POUR ACCROIITRE LES CAPACITÉS À L'EMPLOI ?}

Considérons l'approche par les capacités comme un moyen d'évaluer les effets de la formation sur l'ensemble des opportunités d'emploi qui se présentent aux travailleurs espagnols, lorsqu'ils ont accompli un certain parcours professionnel. Nous n'envisageons pas - comme nous l'avons déjà expliqué précédemment - les effets de la formation sur la période 
d'insertion professionnelle, mais sur les situations d'emploi et de contrat, une fois que l'insertion professionnelle a eu lieu. Rappelons également les limites des données utilisées.

Sur la base de l'ensemble des analyses réalisées, nous pouvons affirmer, en premier lieu, que la situation sur le marché du travail (les fonctionnements) des travailleurs est peu influencée par l'utilisation de ressources formatives. En ce sens, et de façon générale, les ressources formatives à la disposition des travailleurs ne semblent pas améliorer l'ensemble des opportunités d'emploi (les capacités) auxquelles ils ont accès. Nous nuancerons par la suite ce constat, mais nous pouvons affirmer que la formation n'améliore pas les capacités de tous les travailleurs.

En général, la situation d'un travailleur sur le marché du travail espagnol est particulièrement marquée par l'existence d'un marché très flexible pour certains profils. De ce point de vue, nous constatons que, dans la période analysée, certains facteurs de conversion ${ }^{13}$ à caractère institutionnel (segmentation et dualisation du marché du travail espagnol) s'opposent à l'accroissement potentiel des capacités qu'implique le suivi d'un type de formation donné. Ainsi, la formation ne semble pas avoir un effet significatif sur le changement positif de catégorie, le passage d'un CDD à un CDI ou le passage de l'état de chômage à celui d'activité. Ces situations semblent davantage marquées par l'effet de conjoncture de la période analysée que par les effets de la formation.

Toutefois, il semble que cet effet de conjoncture ait interagi positivement, dans les parcours de grande précarité, avec le suivi d'une formation occupationnelle. Le profil de femmes connaissant de nombreuses périodes d'inactivité a vu sa situation d'emploi s'améliorer, et c'est également le profil qui affirme avoir bénéficié de la formation occupationnelle la plus importante.

Parmi les plus jeunes - les travailleurs qui sont arrivés sur le marché du travail après les réformes de la moitié des années $80-$, on note différents types de parcours et d'utilisations associées de la formation. D'une part,

\footnotetext{
13 Rappelons qu'un facteur de conversion est une caractéristique individuelle ou de l'environnement de l'individu qui joue sur les ressources qu'un individu pourrait potentiellement mobiliser et celles qu'il peut réellement utiliser.
}

on trouve un profil de travailleurs qui utilisent peu la formation, mais qui ont néanmoins connu, dans une certaine mesure, une promotion professionnelle au cours de la période. Il s'agit de travailleurs au parcours professionnel précaire, pour lesquels les changements constants d'emploi ou de contrat sont monnaie courante, et dont les capacités seraient étroitement liées aux facteurs structurants du marché du travail et aux stratégies de recrutement des entreprises.

D'autre part, les travailleurs dont le niveau d'étude est le plus élevé ont le plus souvent recours à la formation non formelle, bien que la participation à la formation ne soit pas associée à une augmentation généralisée de l'ensemble des opportunités d'emploi. Parmi ces travailleurs, on distingue deux types de parcours : Le parcours du Travail Temporaire Chronique, dans lequel l'usage intensif de ressources formatives n'augmente pas les capacités. Ces capacités dépendent en effet d'un contexte de flexibilité du cadre réglementaire dont les entreprises tirent profit en se cantonnant aux contrats temporaires ${ }^{14}$; le parcours professionnel, dans lequel la formation continue semble garantir bien plus le maintien sur le poste de travail que la promotion.

Ainsi, la formation (continue et individuelle non formelle) peut sans doute avoir des effets positifs pour certains profils très précis. Cependant, lorsque l'on considère l'ensemble de la population active (ou active à un certain moment de la période analysée), les effets ont un caractère contradictoire. Cela semble indiquer que le type de parcours (nous devrions dire la « logique » du parcours) a un effet qui annule les effets potentiels de la formation. Ainsi, dans une situation de forte flexibilité (en particulier chez certains groupes sociaux, tels que les jeunes ou les femmes), d'autres facteurs semblent plus pertinents pour déterminer la position et les situations des individus sur le marché du travail.

Les paragraphes précédents montrent clairement que les différents types de formation proposés aux travailleurs peinent à lutter contre les inerties dont l'effet causal est beaucoup plus important. Ce sont les

\footnotetext{
14 Bien que, depuis 1996, des subventions et des modalités spécifiques de contrat, à moindres coûts d'embauche, visent à réduire le nombre de contrats temporaires (López Andreu, Lope y Verd, 2007).
} 
circonstances de l'utilisation, et non pas l'utilisation en elle-même, qui confèrent son efficacité à la formation. Cela démontre donc la pertinence du concept senien de « facteur de conversion » dans l'analyse. En ce sens, il nous semble que les résultats obtenus ont le mérite de centrer l'analyse du recours à la formation sur certains parcours individuels déterminés de vie et de travail, qui révèlent l'importance de ce que nous pourrions appeler les « facteurs de conversion liés au parcours ». Des ressources similaires produisent des effets différents (capacités différentes) en fonction de facteurs (structure du marché du travail, législation du travail, politiques de recrutement des entreprises...) qui finissent par générer des parcours professionnels déterminés, difficilement maîtrisables par les individus. Faute de politiques publiques qui interviennent également sur ces facteurs, une augmentation de la formation se traduisant par un accroissement de l'ensemble des opportunités d'emploi nous semble difficile.

Texte traduit de l'espagnol au français par : TSF

\section{Bibliographie}

Alonso Arellano F. (2005), Do trainning programmes get the unemployed back to work? A look at the Spanish experience. Working Paper 05-25, Madrid, Universidad Carlos III.

Alonso L. E. (2007), La crisis de la ciudadanía laboral, Barcelona, Anthropos.

Banyuls J., Miguélez F., Recio A., Cano E., Lorente R. (2009), "The Transformation of the Employment System in Spain: Towards a Mediterranean Neoliberalism ?", en Gerhard Bosch, Steffen Lehndorff y Jill Rubery (eds.): European Employment Models in Flux. A Comparison of Institutional Change in Nine European Countries. New York: Palgrave-Macmillan.

Bonvin J. M., Farvaque N. (2008), Amartya Sen. Une politique de la liberté, Paris, Michalon.

Caprile M., Herrera D., Potrony J., Vallés N. (2008), "La qualitat de l'ocupació a Catalunya", en Montagut, M. (coord.) Societat Catalana 2008, Associació Catalana de Sociologia, Institut d'Estudis Catalans, pp. 97-121.

Caprile M., Potrony J. (2007), La desigualdad en el mercado de trabajo: salario y estabilidad laboral. Ponencia presentada en el IX Congreso Español de Sociología, Barcelona, 13-15/09/2007.
Caprile M., Potrony J. (2010), Mercat de treball $i$ polítiques actives d'ocupació. Barcelona: Fundació Jaume Bofill.

Consejo Económico y Social (2010), Informe sobre Sistema Educativo y Capital Humano. Madrid, Consejo Económico y Social.

Dautrey P. (2007)), « Économie de la connaissance, post-fordisme et savoirs occupationnels en Espagne : une nouvelle donne », Savoirs n 15, pp. 105-120.

Demazière D., Dubar C. (2004), Analyser les entretiens biographiques : l'exemple des récits d'insertion. Québec, Université Laval.

Hernanz Martín V. (2003), El trabajo temporal y la segmentación. Un estudio de las transiciones laborales. Madrid, Consejo Económico y Social.

Homs O., Caprile M., Vallès N., Potrony J. (2006), La qualitat del mercat de treball a Catalunya. Informe elaborado para el Departament de Treball de la Generalitat de Catalunya. Barcelona: CIREM.

López Andreu M., Lope A., Verd J.M. (2007), “El empleo subvencionado: entre las políticas activas de empleo y la mutación del salario", Cuadernos de Relaciones Laborales, Vol. 25, $\mathrm{n}^{\circ}$ 2. pp. 151-178. 
Martín A. (2007), "Les orientacions de les polítiques d'ocupació a Catalunya", a Anuari Societat Catalana 2007, Barcelona, IEC, pp. 01-120.

Mato Diaz F. J. (2002), La formación para el empleo: una evaluación cuasiexperimental. Madrid, Civitas.

Mato Diaz F. J., Cueto Iglesias B. (2008), "Efectos de las políticas de formación a los desempleados", Revista de Economía Aplicada, Vol. XVI, $\mathrm{n}^{\circ} 46$, pp. 61-84.

Merino F. (2008), "Dos o tres itnerarios de Formación Profesional ? Balance y situación actual en España”, Revista Europea de Formación Profesional, vol. 37, $\mathrm{n}^{\circ} 1, \mathrm{pp}$. 55-69.

Planas J. (2005), "El papel de la empresa en la formación de los trabajadores en España", Revista de Educación, n 338, pp. 125-143

Robeyns I. (2003) "Sen's Capability Approach and Gender Inequality: Selecting relevant capabilities", Feminist Economics, vol. 9, n 2-3, pp. 61-92.

Robeyns I. (2005), "The Capability Approach: a theoretical survey". Journal of Human Development and Capabilities: A Multi-Disciplinary Journal for People-Centered Development, Vol. 6, $\mathrm{n}^{\circ} 1$, pp. 93-117.

Salais R., Villeneuve, R. (2004), Europe and the Politics of Capabilities. Cambridge, Cambridge University Press.
Santos A., Poveda M. (2002), Trabajo, empleo y cambio social. Valencia, Tirant lo Blanch. $2^{\mathrm{a}}$ edición.

Segura J., Durán F., Toharia L. y Bentolila S. (1991), Análisis de la contratación temporal en España. Madrid, Ministerio de Trabajo y Seguridad Social.

Sen A. (1993), "Capability and Well-Being”, en Amartya K. Sen y Martha Nussbaum (eds.), The Quality of Life. Oxford: Oxford University Press.

Sen A. (1999), Commodities and Capabilities. Oxford, Oxford University Press.

Sen A. (2002), "Introduction: Rationality and Freedom", en Amartya Sen, Rationality and Freedom, Cambridge, Massachusetts: Belknap Press.

Sen A. (2006), "What do we want from a theory of justice ?", The Journal of Philosophy, vol. 103, n 5 , pp. 215-238.

Toharia L., Malo M. (2000), “The Spanish experiment: pros and cons of flexibility at the margin", en Esping-Andersen, G. y Regini, M. (eds.): Why deregulate labour markets?, Oxford: Oxford University.

Verd J.M., Vero J., López Andreu M. (2009), "Trayectorias laborales y enfoque de las capacidades: elementos para uan evaluación longitudinal de las políticas de protección social", Sociología del Trabajo, $\mathrm{n}^{\circ} 167$, pp. 127-150. 


\section{Résumé}

\section{Les effets de la formation diffèrent selon les parcours sur le marché du travail : l'exemple catalan}

Martí López-Andreu, Joan Miquel Verd

Le présent article tente, pour les personnes déjà insérées sur le marché du travail, d'analyser l'impact de la formation non formelle ou de l'accroissement du niveau d'études sur les changements de situation sur le marché du travail (emploi ou chômage), les changements de contrat de travail ou de catégorie professionnelle. Les effets de la formation sont analysés à l'aune des différents types de parcours observés sur le marché du travail en Catalogne. En effet, les profondes réformes allant dans le sens de la flexibilité, introduites en Espagne depuis 1985, ont généré divers profils de travailleurs présentant des parcours très différenciés. Les résulats de nos travaux montrent que les caractéristiques sociales de ces parcours semblent annuler, dans certains cas, les effets positifs potentiels de la formation suivie.

\section{Mots clés :}

Situation du marché du travail, cheminement professionnel, formation des adultes, formation des chômeurs, flexibilité du marché du travail, Catalogne, Espagne

Journal of Economic Literature: J 24, J 62 